\title{
NOVEL ANALYSIS SCHEME TO ANALYZE MULTILAYER DIELECTRIC MICROSTRIP ANTENNAS
}

\author{
S. Sriram, Student Member, IEEE and T. S. Vedavathy
}

Electrical Communication Engineering Department, Indian Institute of Science, Bangalore, INDIA.

\begin{abstract}
An efficient technique utilizing the advantages of the Conformal Mapping Technique and the Cavity Model has been proposed in this paper to analyze easily and effectively, the multilayer stacked microstrip antenna structures. The proposed method is generalized to be suitable in analyzing such microstrip antennas of various geometries, namely, the rectangular, circular disc, annular ring and triangular patches. The closed form expressions for the resonant frequency, for any mode of operation, involve simple calculations yielding results which agree well with the experimental values. This unique method solves the long-lasting problem of analyzing multilayer stacked microstrip antennas of various geometries with ease.
\end{abstract}

\section{INTRODUCTION}

The numerous advantages of microstrip antennas in being low profile, compact, conformal, cost-effective, etc. find it best suited for various applications. The basic requirement of the design engineer includes the proper choice of the structure and the proper geometry, which would in turn demand a proper analysis technique which predicts accurately the behavior of the antenna under consideration, viz., the resonant frequency, the impedance, etc

The single layer dielectric microstrip antenna suffers from a narrow bandwidth. which restricts the range of applications. To combat this problem, the simplest approach would be to increase the thickness of the substrate, but this has other disadvantages. The best alternative is by using multilayer-stacked structures. Now, the issue is to analyze effectively and easily the stacked structures. There are a few models suggested in the literature. of which the cavity model helps in determining the resonant frequency of simpler structures, extending upto structures involving air-gaps. Besides the need to find some simple technique for effective analysis of such structures. it also requires the method to be general to apply to several geometries of patch antennas, multidielectric structures, with and without dielectric cover. The proposed method successfully aims at meeting all these requirements with simplicity and effectiveness.

\section{FORMULATION}

\section{A. Conformal Mapping Technique (CMT)}

The main advantages of the CMT are its simplicity and close approximation besides involving simpler functions, rather than tedious integrals involved in other techniques. The conformal mapping of a boundary between dielectrics is valid since it retains the angles of refraction of the electric field at the boundary, while maintaining the electrical properties of the geometry in the mapped plane. This property helps in determining the capacitance (or effective dielectric constant) of the transformed simpler geometry and hence its resonant frequency.

The proposed method presents a simple scheme to transform any regular geometry of patch antenna to a rectangular geometry of appropriate dimensions. This transformed rectangular geometry of length $L$ and width $W$ is then mapped from one complex plane to another by proper set of transformations which results in a simpler structure for which the effective dielectric constant is evaluated (Fig. 1).

\section{B. Cavity Model}

The cavity model is sought to deternine the characteristic equation of the given regular geometry of analysis. The characteristic equation is then solved to determine the eigen values of the particular $\mathrm{TM}_{\mathrm{nm}}$ mode of operation. This ensures the proposed method to form a general technique as a closed form approach to calculate the resonant frequencies of any mode of multidielectric layer antennas of any given geometry. 


\section{ANALYSIS AND DESIGN}

The resonant frequency of a given microstrip patch antenna is determined from the basic formula:

$$
f_{n m}=\frac{c}{\lambda \sqrt{\varepsilon_{e f f}}}
$$
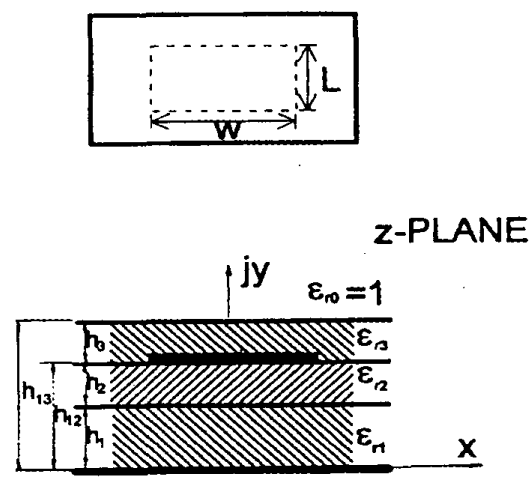

(a)
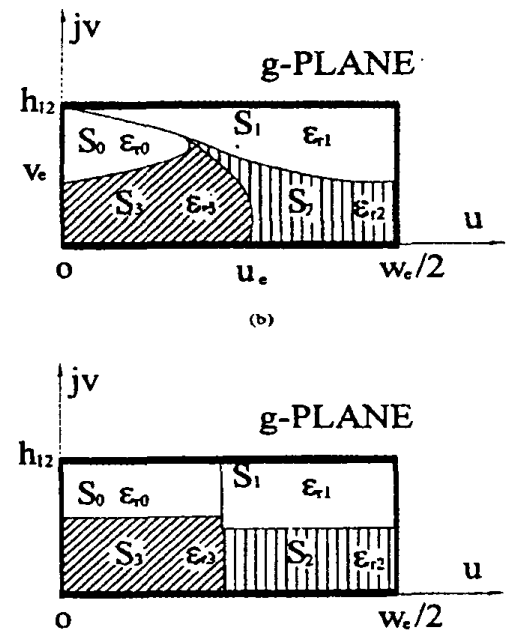

'(c)

Fig.1. A multilayer dielectric rectangular microstrip antenna and its transformations [1]

where $c$ is the velocity of light in free space, $\lambda$ is the wavelength of operation and $\varepsilon_{\text {eff }}$ is the effective dielectric constant.

\section{A. Rectangular Patch Antenna}

A rectangular patch antenna of length. $L$ and width. $W$ is analyzed to determine the resonant frequency using CMT [1] as given in Fig. 1. with calculations of effective dielectric constant, $\varepsilon_{\text {eff }}$ and effective length and width. The formula presented in [1] determines the resonant frequency of the rectangular patch for only the fuindamental mode $\mathrm{TM}_{10}$. Now, using the cavity model which gives the appropriate eigen values of various $\mathrm{TM}_{\mathrm{nm}}$ modes, (1) is modified to determine the resonant frequency of any $\mathrm{TM}_{\mathrm{nm}}$ mode of a rectangular patch antenna as given below:

$$
f_{n m}=\frac{k_{n m} c}{2 \pi \sqrt{\varepsilon_{e f f}}}
$$

where, the eigen values, $k_{r m}$ for a rectangular patch antenna are given by:

$$
k_{n m}=\sqrt{\left[\frac{n \pi}{L}\right]^{2}+\left[\frac{m \pi}{W}\right]^{2}}
$$

which implies

$$
f_{r m}=\frac{k_{r n n}^{\prime} c}{2 L \sqrt{\varepsilon_{e f f}}}
$$

where the modified eigen values, $k_{n m}^{\prime}$ are calculated from

$$
k_{n m}^{\prime}=\sqrt{n^{2}+\left[\frac{m L}{W}\right]^{2}}
$$

Now by suitably replacing $L$ and $W$ by their effective dimensions determined by the procedure in [1]. one can predict the resonant frequency of any mode of operation for a rectangular patch antenna.

\section{B. Triangular Patch Antenna}

The analysis of an equilateral triangle patch antenna of side ' $a$ ' is done by first transforming this geometry into a rectangular geometry of appropriate dimensions, which correspond to:

$$
W=\frac{a}{2}
$$




$$
L=\frac{\sqrt{3} a}{2}
$$

For this structure, the effective dielectric constant is determined and the modified eigen value equation is used to determine the eigen value for the particular $\mathrm{TM}_{\mathrm{mn}}$ mode of interest.

$$
k_{n m}^{\prime}=\frac{2}{\sqrt{3}} \sqrt{m^{2}+n^{2}+m n}
$$

Then, substituting (6)-(8) in (4), the resonant frequency of the mode of interest of the triangular patch antenna can be evaluated. The effective dimensions are determined by incorporating $a_{e f f}$ instead of $a$, in the above formulas as specified in [2].

\section{Circular Disc and Annular Ring Antennas}

The disc and annular ring geometries come under the circular patch configuration. An annular ring microstrip antenna with an inner radius of ' $a$ ' and outer radius of ' $b$ ' units is first transformed into a rectangular patch of dimensions given below:

$$
\begin{gathered}
W=b-a \\
L=\frac{\pi(b+a)}{2}
\end{gathered}
$$

Now for the transformed structure, the effective dielectric constant is determined and the modified eigen values are obtained by solving the following characteristic equation

$$
J_{n}^{\prime}\left(k_{n m}^{\prime} a\right) Y_{n}^{\prime}\left(k_{n m}^{\prime} b\right)-J_{n}^{\prime} k_{n m}^{\prime}\left(k_{n m}^{\prime} b\right) Y_{n}^{\prime}\left(k_{n m}^{\prime} a\right)=0
$$

where $J_{n}$ and $Y_{n}$ are the Bessel function of order $\mathrm{n}$ of first and second kind respectively.

The same set of values applies for a circular disc wherein the inner radius tends to zero. Then, the values of $W, L$ and $k_{n m}^{\prime}$ are substituted in (4) and the resonant frequency of the mode of operation of a circular disc/ring antenna can be determined. The effective radii of the circular antennas are determined by the formulas given in (28)-(29) of [3].

\section{Multidielectric laver Stacked Antenna}

The closed form expression thus derived using CMT is useful to determine upto two layer dielectric (of different substrates) with a dielectric cover (single layer). The same procedure can be applied to multilayer substrates and/or multilayer superstrates (dielectric cover over the radiating patch) by taking 2 layers from the radiating patch at a time and calculating the effective dielectric constant due to those two layers. Then. these two layers are now replaced by a single layer of effective dielectric constant and the total thickness. This procedure is continued till the ground plane is reached on the substrate section and free space on the superstrate section.

\section{RESILTS AND DISCUISSION}

The extent of application and usefulness of the proposed method is clearly evident; it is used to determine the resonant frequencies of various structures and a comparison is made with the experimental results published in the literature.

\section{A. Rectangular Microstrip Antenna}

The rectangular geometry analyzed in [1] has dimensions of length, $L=3.325 \mathrm{~cm}$; width, $W=3.225 \mathrm{~cm}$; substrate thickness $=0.318 \mathrm{~mm}$; and both substrate and superstrate dielectric constant of 2.32. The resonant frequencies of the dominant mode with varying thicknesses of the dielectric cover are tabulated in Table $I$.

\section{TABLE I}

RESONANT FREQUENCIES OF RECTANGILAR PATCH ANTENNA WITH VAR YING DIELECTRIC COVER THICKNESS

\begin{tabular}{|c|c|c|}
\hline $\begin{array}{c}\text { Cover } \\
\text { Thickness } \\
(\mathrm{cm})\end{array}$ & $\begin{array}{c}\text { Calc. } \\
(\mathrm{GHz})\end{array}$ & $\begin{array}{c}\text { Meas. } \\
(\mathrm{GHz})\end{array}$ \\
\hline 0.318 & 2.716 & 2.749 \\
\hline 0.636 & 2.699 & 2.704 \\
\hline 0.954 & 2.687 & 2.682 \\
\hline 1.272 & 2.678 & 2.670 \\
\hline
\end{tabular}

\section{B. Triangular Microstrip Antenna}

An equilateral triangular patch of side, $a=10 \mathrm{~cm}$; substrate thickness of $0.159 \mathrm{~cm}$ has been analyzed and the values are compared in Table II. 
TABLE II

RESONANT FREOLENCIES OF EQUILATERAL TRIANGULAR MICROSTRIP ANTENNA

\begin{tabular}{|c|c|c|c|c|}
\hline \multirow{2}{*}{ Mode } & \multicolumn{2}{|c|}{$\varepsilon_{\mathrm{r}}=2.32$} & \multicolumn{2}{c|}{$\varepsilon_{\mathrm{r}}=9.8$} \\
\cline { 2 - 5 } & Calc. & Meas. & Calc. & Meas. \\
\hline $\mathrm{TM}_{10}$ & 1.269 & 1.280 & 0.638 & 0.64 \\
\hline $\mathrm{TM}_{11}$ & 2.192 & 2.242 & 1.104 & 0.90 \\
\hline $\mathrm{TM}_{20}$ & 2.529 & 2.550 & 1.273 & 1.28 \\
\hline $\mathrm{TM}_{21}$ & 3.339 & 3.40 & 1.682 & 1.66 \\
\hline $\mathrm{TM}_{30}$ & 3.783 & 3.824 & 1.906 & 1.91 \\
\hline
\end{tabular}

\section{Circular Disc Antenna}

A circular disc of radius $5 \mathrm{~cm}$, with a dielectric substrate of $\varepsilon_{r}=2.32$ and thickness of $0.159 \mathrm{~cm}$ has been considered. An airgap is introduced between the ground plane and the dielectric substrate. Table III lists out the resonant frequencies calculated by the proposed method which is in agreement with the experimental values [4].

TABLE III

RESONANT FREQUENCIES OF CIRCULAR DISC MICROSTRIP ANTENNA

\begin{tabular}{|c|c|c|c|c|c|c|}
\hline $\begin{array}{c}\text { Airgap } \\
(\mathrm{cm})\end{array}$ & \multicolumn{2}{|c|}{0} & \multicolumn{2}{c|}{0.05} & \multicolumn{2}{c|}{0.1} \\
\hline Mode & Calc. & Meas. & Calc. & Meas. & Calc. & Meas. \\
\hline $\mathrm{TM}_{11}$ & 1.147 & 1.128 & 1.286 & 1.286 & 1.359 & 1.350 \\
\hline $\mathrm{TM}_{21}$ & 1.898 & 1.879 & 2.129 & 2.136 & 2.249 & 2.256 \\
\hline $\mathrm{TM}_{31}$ & 2.606 & 2.596 & 2.924 & 2.951 & 3.089 & 3.106 \\
\hline
\end{tabular}

\section{Annular Ring Microstrip Antenna}

An annular ring of inner radius $3.5 \mathrm{~cm}$ and outer radius $7.0 \mathrm{~cm}$. with a dielectric substrate of $\varepsilon_{r}=2.32$ and thickness of $0.159 \mathrm{~cm}$ has been considered. An airgap is introduced between the ground plane and the dielectric substrate. Table IV lists out the resonant frequencies calculated by the proposed method which are in agreement with the experimental values [3].

\section{TABLE IV}

RESONANT FREQUENCIES OF ANNULAR RING MICROSTRIP ANIENNA

\begin{tabular}{|c|c|c|c|c|c|c|}
\hline $\begin{array}{c}\text { Airgap } \\
\text { (cm) }\end{array}$ & \multicolumn{2}{|c|}{0} & \multicolumn{2}{c|}{0.05} & \multicolumn{2}{c|}{0.1} \\
\hline Mode & Calc. & Meas. & Calc. & Meas. & Calc. & Meas. \\
\hline TM11 & 0.622 & 0.626 & 0.703 & 0.720 & 0.748 & 0.778 \\
\hline TM21 & 1.226 & 1.229 & 1.383 & 1.415 & 1.470 & 1.516 \\
\hline TM31 & 1.798 & 1.800 & 2.027 & 2.075 & 2.151 & 2.212 \\
\hline TM12 & 2.822 & 2.757 & 3.127 & 3.040 & 3.273 & 3.240 \\
\hline
\end{tabular}

Note: (alc. and leas. in the table entries correspond to the Calculated and leasured Values of the Resonont Frequencies (which are in $(\mathrm{iHz})$

While the cavity model analyses effectively only simpler microstrip antemna structures and those with only air-gap, the proposed model presents a simple effective method to analyze multilayer dielectric structure with different dielectric substrates, and with multiple layers of dielectric cover, very efficiently and accurately.

\section{CONCLUSION}

The paper presents the effective utilization of Conformal Mapping Technique to analyze the multilayer microstrip antennas of various geometries to derive the resonant frequency of all the modes. This is achieved by using the roots of the characteristic equation as proposed by cavity model. As CMT is very useful in analyzing the multilayer dielectric structure, which is otherwise complex, the problem simplifies to obtaining the effective dielectric constant of the patch using CMT. Hence a simple closed form expression for obtaining the frequency of resonance of any mode is derived. The values thus calculated agree well with the experimental values and are validated with those published in the literature. The paper presents the analysis of rectangular, triangular, circular disc and annular ring patch antennas by CMT and Cavity Model. The proposed scheme gives ample scope to analyze multilayer multiconductor stacked structures. with great simplicity and ease of calculation. This gives a comprehensive solution to the long-lasted requirement of a simpler technique to analyze complex stacked structures efficiently.

\section{REFERENCES}

[1] SHUN-SHI ZHONG, GANG LIU and GHULAM QASIM : 'Closed form expressions for resonant frequency of rectangular patch antennas with multidielectric layers', IEEE Trans., 1994, AP-42, (9). pp.1360-1363.

[2] KERIM GÜNEY: 'Comments on "On the Resonant Frequencies of Microstrip Antennas" . IEEE Trans., 1994. AP-42, (9). pp. 1363-1365.

[3] LEE, K. F. and DAHELE. J. S.: The two-layered annular-ring microstrip antenna'. Int. J. Electronics, 1986. 61, (2). pp. 207-217.

[4] DAHELE, J. S. and LEE K. F.: 'Theory and experiment on microstrip antennas with airgaps'. IEE Proc. Pt. $H, 1985,132,(7)$, pp. $455-460$ 